Pacific Journal of Mathematics

SURJECTIVE EXTENSION OF THE REDUCTION OPERATOR 


\title{
SURJECTIVE EXTENSION OF THE REDUCTION OPERATOR
}

\section{Moses GlasNer aNd Mitsuru NAKaI}

\begin{abstract}
In this paper it is shown that there exists a Riemann surface $R$ and a nonnegative 2 -form $P$ on $R$ such that the space of energy finite solutions of $d * d u=u P$ on $R$ is properly contained in the space of Dirichlet finite solutions yet the subspaces of bounded functions in these two spaces coincide.
\end{abstract}

Consider a nonnegative locally Hölder continuous 2-form $P$ on a hyperbolic Riemann surface $R$. Let $P X(R)$ denote the space of solutions of $d * d u=u P$ on $R$ satisfying a certain boundedness property $X$, e.g. $D$ (finite Dirichlet integral $\int_{R} d u \wedge * d u$ ), $E$ (finite energy integral $\int_{R} d u \wedge$ $* d u+u^{2} P$ ), $B$ (finite supremum norm) or the combinations $B D$ and $B E$. The reduction operator $T_{X}$ is defined to be the linear injection of the space $P X(R)$ into the space $H X(R)$ such that for each $u \in P X(R)$ there is a potential $p_{u}$ on $R$ with $\left|u-T_{X} u\right| \leq p_{u}$. The unique existence of $T_{X}$ for the cases $X=B, D, E$ was established in [5] together with the representations

$$
T_{X} u(z)=u(z)+\frac{1}{2 \pi} \int_{R} G_{R}(z, \zeta) u(\zeta) P(\zeta)
$$

where $G_{R}(\cdot, \zeta)$ is the Green's function for $T$ with pole at $\zeta$.

One of the central questions concerning reduction operators is whether

$$
T_{B X} \text { is surjective implies that } T_{X} \text { is surjective, }
$$

$X=D, E$. Since $P B X(R)$ is dense in $P X(R)$ in the same fashion as $H B D(R)$ is dense in $H D(R)$ (cf. [1], [4]), it is natural to conjecture that the implication (1) holds. Surprisingly, in [12] and [7] it was shown that (1) is false for $X=D, E$. Even the stronger conditions $\int_{R} P<+\infty$, $\int_{R \times R} G_{R}(z, \zeta) P(z) P(\zeta)<+\infty$ do not imply the surjectiveness of $T_{E}$ and $T_{D}$ respectively as was shown in [8], [9], [10].

In this connection we raise the question whether the fact that (1) does not hold for $X=E$ by itself implies that (1) does not hold for $X=D$. This is closely related to the following: Is it true that $P B D(R)=P B E(R)$ implies that $P D(R)=P E(R)$ ? We shall show here that the answer to the latter question is no even under the stronger assumption that $P B D(R)=$ $P B E(R) \cong H B D(R)$ which is a consequence of the surjectiveness of $T_{B E}$. Therefore the former question will also be settled in the negative. 
1. We freely use the machinery of the Royden ideal boundary theory and the equation $d * d u=u P$, as well as some of the techniques introduced in [2]. Let $W \subset R$ be an open set with $\partial W$ consisting of analytic curves. The extremization operator $\mu_{x}^{P}, X=D, E, B D, B E$, is the linear injection of the relative class $P X(W ; \partial W)$ into $P X(R)$ characterized by the property that for each $u \in P X(W ; \partial W)$ there is a potential $p_{u}$ on $R$ with $\left|u-\mu_{X}^{P} u\right| \leq p_{u}$. An alternate characterization of $\mu_{X}^{P}$ is the condition that $u-\mu_{X}^{P} u \mid \Delta=0$ for each $u \in P X(W ; \partial W)$. We also consider the reduction operator $T_{X, W}: P X(W) \cap \tilde{M}(R) \rightarrow H D(W)$, which can be characterized by the condition $u-T_{X, W} u \mid \partial W \cup b W=0$, where $b W=$ $(\bar{W} \cap \Delta) \backslash \overline{\partial W}$. In particular, $T_{X, W}: \stackrel{P}{P}(W ; \partial W) \rightarrow H D(W ; \partial W)$ is determined by $u-T_{X, W} u \mid b W=0$.

In [2] we completely characterized the functions in $H D(R)$ that are in the image of $\mu_{D}$. In view of the following result, which we will make essential use of here, the corresponding problem for $P X(R)$ is also settled.

THEOREM. Let $u$ be in $P X(R)$ and set $h=T_{X} u$. Then $u \in$ $\mu_{X}^{P}(P X(W ; \partial W))$ if and only if $h \in \mu_{D}(H D(W ; \partial W)), X=D$ or $E$.

The necessity is simple. Let $s \in P X(W ; \partial W)$ such that $\mu_{X}^{P} s=u$. Define $v=T_{X, W} s$. Since $v|\Delta=s| \Delta=u|\Delta=h| \Delta$, we conclude that $h=\mu_{D} v$. Conversely, assume that $h=\mu_{D} v, v \in H D(W ; \partial W)$. It suffices to establish the sufficiency in case $v \geq 0$. We begin by showing that the assertion holds for $X=D$. For each positive integer $k$, set $\psi_{k}=(h \cap k)$ $\cup k^{-1}$ and $v_{k}=\Pi_{\overline{R \backslash W}}\left(\psi_{k}-k^{-1}\right)$, the harmonic projection of $\psi_{k}-k^{-1}$ on $W$. The sequence $\left\{v_{k}\right\}$ is easily seen to have the following properties (cf. [2]): $v_{k} \in H B D(W ; \partial W), v_{k} \leq v_{k+1} \leq v, v=C D-\lim v_{k}, \operatorname{Supp}\left(v_{k} \mid \Delta\right) \subset$ $\{p \in \Delta \mid v(p)>0\}, \lim \left(v_{k} \mid \Delta\right)=v \mid \Delta$ and

$$
D_{W}\left(v_{k}\right) \leq D_{W}(v) \text {. }
$$

In view of $v|\Delta=h| \Delta=u \mid \Delta$ and $u \in P D(R)$ we have that $v \mid \Delta \backslash \Delta_{P}=0$. Thus $\operatorname{Supp}\left(v_{k} \mid \Delta\right) \subset b W \cap \Delta_{P}$ and consequently there is a function $s_{k} \in P B D(W ; \partial W)$ such that $s_{k}\left|\Delta=v_{k}\right| \Delta$. By the maximum principle, $s_{k} \leq s_{k+1} \leq v_{k+1} \leq v$ on $R$ and then by the Harnack principle $s=C-\lim s_{k}$ exists on $W$. Since $v \mid R \backslash W=0$, we actually have $s=C$-lim $s_{k}$ on $R$ with $s \mid R \backslash W=0$, in particular, $s \in P(W ; \partial W)$.

We show that in fact $s \in P D(W ; \partial W)$. In view of the identity $D_{W}(u)=D_{W}\left(T_{D, W} u\right)+\langle u, u\rangle_{W}^{P}$ (cf. [5]), we have $\langle u, u\rangle_{W}^{P}<+\infty$. Comparing boundary values shows that $s_{k} \leq u$ on $W$. This together with (3) gives the following bound on $D_{W}\left(s_{k}\right)$ :

$$
D_{W}\left(s_{k}\right)=D_{W}\left(v_{k}\right)+\left\langle s_{k}, s_{k}\right\rangle_{W}^{P} \leq D_{W}(v)+\langle u, u\rangle_{W}^{P}<+\infty .
$$

By Fatou's lemma we obtain $D_{W}(s)<+\infty$. 
In view of $s \leq u$, we have $s|\Delta \leq u| \Delta$. On the other hand,

$$
s\left|\Delta \geq \lim \left(s_{k} \mid \Delta\right)=\lim \left(v_{k} \mid \Delta\right)=v\right| \Delta=u \mid \Delta .
$$

We conclude that $\mu_{D}^{P} s=u$, establishing the sufficiency in case $X=D$. If in addition $u \in P E(R)$, then $s \leq u$ gives $\int_{W} s^{2} P \leq \int_{W} u^{2} P<+\infty$, i.e. $s \in P E(W ; \partial W)$, which completes the proof.

2. Let $T$ be a hyperbolic Riemann surface such that $H B D(R)$ consists only of the constant functions. Examples of such surfaces are the Tôki surface and the Tôki covering surfaces (cf. [11]). The harmonic boundary of $T$ consists of a single point which we denote by $p^{*}$. Fix $q_{0} \in T$ and consider the polar coordinate differentials $(d r, d \theta)$ on $T$ defined by

$$
\frac{d r(z)}{r(z)}=-d G_{T}\left(z, q_{0}\right), d \theta(z)=-* d G_{T}\left(z, q_{0}\right)
$$

Fix $\alpha$ such that $\bar{U}_{\alpha}$ is homeomorphic to a closed disk, where $U_{\alpha}=$ $\left\{p \in T \mid r(p)<e^{-\alpha}\right\}$. Also fix $\beta \leq \alpha / 2$. For each $\lambda>0$ define a rotation free 2-form $P_{\lambda}=\varphi_{\lambda}(r) r d r \wedge d \theta$, where $\varphi_{\lambda}$ is the nonnegative Hölder continuous function on $[0,1)$ determined by the following conditions:

$$
\varphi_{\lambda}(t)= \begin{cases}(1-t)^{-\lambda}, & \text { if } e^{-\beta} \leq t<1, \\ \text { linear, } & \text { if } e^{-2 \beta} \leq t<e^{-\beta} \\ 0, & \text { if } 0 \leq t<e^{-2 \beta}\end{cases}
$$

According to the main results of [6] we have

$$
\begin{aligned}
& P_{\lambda} B D(T)=\{0\} \text { if and only if } \lambda \in[3 / 2,+\infty), \\
& P_{\lambda} B E(T)=\{0\} \text { if and only if } \lambda \in[1,+\infty) .
\end{aligned}
$$

By our choice of $T$ here it follows that $\operatorname{dim} P_{\lambda} X(T)=\operatorname{dim} P_{\lambda} B X(T) \leq 1$ for $X=D, E$. Furthermore, it can be seen from [6] that

$$
\begin{gathered}
\int_{T} G_{T}\left(q_{0}, \cdot\right) P_{\lambda}<+\infty, \text { if } \lambda<2, \\
\langle 1,1\rangle_{T}^{P_{\lambda}}<+\infty, \text { if } \lambda<\frac{3}{2}, \\
\int_{T} P_{\lambda}<+\infty, \text { if } \lambda<1 .
\end{gathered}
$$

Set $W_{\alpha}=T \backslash \bar{U}_{\alpha}$. Then $P_{\lambda} X\left(W_{\alpha} ; \partial W_{\alpha}\right)=P_{\lambda} B X\left(W_{\alpha} ; \partial W_{\alpha}\right)$ is isometric to $P_{\lambda} X(T)=P_{\lambda} B X(T), X=D, E$. So for each $\lambda \in(0,1]$ we may choose $w_{\lambda} \in P_{\lambda} D\left(W_{\alpha} ; \partial W_{\alpha}\right)$ with $w_{\lambda}\left(p^{*}\right)=1$. Then $w_{\lambda}$ spans $P_{\lambda} D\left(W_{\alpha} ; \partial W_{\alpha}\right)$. For 
$\lambda \in(0,1), w_{\lambda} \in P_{\lambda} E\left(W_{\alpha} ; \partial W_{\alpha}\right)$ whereas $\int_{W_{\alpha}} w_{1}^{2} P_{1}=+\infty$. Define

$$
\Phi(\lambda)=E_{T}^{P_{\lambda}}\left(w_{\lambda}\right) \text {. }
$$

LEMMA. $\Phi$ is a continuous function on $(0,1)$ and $\liminf _{\lambda \uparrow 1} \Phi(\lambda)=+\infty$.

Fix $\lambda_{0} \in(0,1)$. We first show that $\Phi$ is continuous from the right at $\lambda_{0}$. Let $\lambda_{0} \leq \lambda<\nu<1$. Since $P_{\lambda} \leq P_{\nu}$, we see that $w_{\lambda}$ is a supersolution with respect to $d * d u=u P_{\nu}$ and thus $w_{\nu} \leq w_{\lambda} \leq w_{\lambda_{0}}<1$. The function $\hat{w}=\lim _{\lambda \downarrow \lambda_{0}} w_{\lambda}$ exists and $\hat{w} \leq w_{\lambda_{0}}$ on $T$. The Harnack inequality applied to (4) implies $\int_{T} G_{T}(\zeta, \cdot) P_{1}<+\infty$, for any $\zeta \in W_{\alpha}$ and hence $\int_{W_{\alpha}} G_{W_{\alpha}}(\zeta, \cdot) P_{1}<+\infty$. Set

$$
\tau^{P_{\lambda}} \varphi(\zeta)=\frac{1}{2 \pi} \int_{W_{\alpha}} G_{W_{\alpha}}(\zeta, z) \varphi(z) P_{\lambda}(z),
$$

for a suitable function $\varphi$ on $W_{\alpha}$. Since $w_{\lambda} P_{\lambda}<P_{1}$ on $W_{\alpha}$, the Lebesgue dominated convergence theorem gives $\lim _{\lambda_{\downarrow} \lambda_{0}} \tau^{P_{\lambda}} w_{\lambda}=\tau^{P_{\lambda_{0}}} \hat{w}$. Note that for any $\lambda, T_{D, W_{\alpha}} w_{\lambda}$ is the same function $v \in H B D\left(W_{\alpha} ; \partial W_{\alpha}\right)$. Therefore,

$$
\begin{aligned}
v & =w_{\lambda_{0}}+\tau^{P_{\lambda_{0}} w_{\lambda_{0}}} \geq \hat{w}+\tau^{P_{\lambda_{0}} \hat{w}} \\
& =\lim _{\lambda \uparrow \lambda_{0}}\left(w_{\lambda}+\tau^{P_{\lambda}} w_{\lambda}\right)=v
\end{aligned}
$$

on $W_{\alpha}$. This implies that $w_{\lambda_{0}}=\hat{w}$ on $W_{\alpha}$ and consequently on $T$. By Dini's theorem we arrive at $w_{\lambda_{0}}=B$ - $\lim _{\lambda \uparrow \lambda_{0}} w_{\lambda}$ on $T$.

We continue with $\lambda_{0} \leq \lambda<\nu<1$. Note that the function $w_{\lambda}-w_{\nu}$ is $P_{\lambda}$ energy finite. Indeed, $w_{\lambda}-w_{\nu}$ is clearly Dirichlet finite and the inequality $0 \leq w_{\lambda}-w_{\nu} \leq w_{\lambda}$ gives $\int_{T}\left(w_{\lambda}-w_{\nu}\right)^{2} P_{\lambda} \leq \int_{T} w_{\lambda}^{2} P_{\lambda}<+\infty$. Since $w_{\lambda}-w_{\nu}$ vanishes at $p^{*}$ we may choose a sequence $\left\{f_{n}\right\} \subset M_{0}(T)$ such that $w_{\lambda}-w_{\nu}=B E^{P_{\lambda}-\lim } f_{n}$. Moreover, the sequence $\left\{f_{n}\right\}$ may be chosen with $f_{n} \mid U_{\alpha}=0$ since $w_{\lambda}-w_{\nu}$ has this property. Thus

$$
E_{T}^{P_{\lambda}}\left(w_{\lambda}-w_{\nu}, w_{\lambda}\right)=\lim _{n} E_{W_{\alpha}}^{P_{\lambda}}\left(f_{n}, w_{\lambda}\right)=0
$$

and consequently

$$
\begin{aligned}
0 & \leq D_{T}\left(w_{\nu}-w_{\lambda}\right) \leq E_{T}^{P_{\lambda}}\left(w_{\nu}-w_{\lambda}\right) \\
& =E_{T}^{P_{\lambda}}\left(w_{\nu}\right)-E_{T}^{P_{\lambda}}\left(w_{\lambda}\right) \leq \Phi(\nu)-\phi(\lambda) .
\end{aligned}
$$

This shows that $\lim _{\lambda \downarrow \lambda_{0}} \Phi(\lambda)$ exists which in turn implies that $\left\{w_{\lambda}\right\}$ is $D$-Cauchy. By Kawamura's lemma we arrive at $w_{\lambda_{0}}=B D-\lim _{\lambda \downarrow \lambda_{0}} w_{\lambda}$, and in particular, $\lim _{\lambda \downarrow \lambda_{0}} D_{T}\left(w_{\lambda}\right)=D_{T}\left(w_{\lambda_{0}}\right)$. By (6), $\int_{T} P_{\nu}<+\infty$ and we apply the Lebesgue dominated convergence theorem to obtain $\lim _{\lambda \downarrow \lambda_{0}} \int_{T} w_{\lambda}^{2} P_{\lambda}=\int_{T} w_{\lambda_{0}}^{2} P_{\lambda_{0}}$. This completes the proof of $\lim _{\lambda \downarrow \lambda_{0}} \Phi(\lambda)=$ $\Phi\left(\lambda_{0}\right)$. 
We now consider $\lambda_{0} \in(0,1]$ and show that $w_{\lambda_{0}}=B D-\lim _{\lambda_{\uparrow} \lambda_{0}} w_{\lambda}$. Let $0<\nu<\lambda \leq \lambda_{0}$ and note that $w_{\lambda_{0}} \leq w_{\lambda}<w_{\nu}<1$. Thus $\lim _{\lambda \uparrow \lambda_{0}} w_{\lambda}$ exists and by an argument analogous to the one above we see that actually $w_{\lambda_{0}}=B-\lim _{\lambda_{\uparrow} \lambda_{0}} w_{\lambda}$. Since $w_{\nu}-w_{\lambda}$ vanishes at $p^{*}$ we can find a sequence $\left\{f_{n}\right\} \subset M_{0}(T)$ such that $w_{\nu}-w_{\lambda}=B D$-lim $f_{n}$. We choose $\left\{f_{n}\right\}$ with the additional properties $f_{n} \geq 0, f_{n} \mid U_{\alpha}=0$. Thus

$$
\begin{aligned}
D_{T}\left(w_{\nu}-w_{\lambda}, w_{\nu}\right) & =\lim _{n} D_{W_{\alpha}}\left(f_{n}, w_{\nu}\right) \\
& =-\lim _{n} \int_{W_{\alpha}} f_{n} d * d w_{\nu} \leq 0,
\end{aligned}
$$

which implies that

$$
0 \leq D_{T}\left(w_{\lambda}-w_{\nu}\right) \leq D_{T}\left(w_{\lambda}\right)-D_{T}\left(w_{\nu}\right) .
$$

Thus $D_{T}\left(w_{\lambda}\right)$ increases as $\lambda$ increases and is bounded above by $D_{T}\left(w_{\lambda_{0}}\right)$. Therefore $\left\{w_{\lambda}\right\}$ is $D$-Cauchy and by Kawamura's lemma $w_{\lambda_{0}}=$ $B D-\lim _{\lambda \uparrow \lambda_{0}} w_{\lambda}$.

In case $\lambda_{0} \in(0,1)$, as before we see that $\lim _{\lambda \uparrow \lambda_{0}} \int_{T} w_{\lambda}^{2} P_{\lambda}=\int_{T} w_{\lambda_{0}}^{2} P_{\lambda_{0}}$. We arrive at $\lim _{\lambda \uparrow \lambda_{0}} \Phi(\lambda)=\Phi\left(\lambda_{0}\right)$ and the continuity of $\Phi$ at $\lambda_{0}$ is established. In case $\lambda_{0}=1$ we apply Fatou's lemma to conclude that $+\infty=\int_{T} w_{1}^{2} P_{1} \leq \liminf _{\lambda \uparrow 1} \int_{T} w_{\lambda}^{2} P_{\lambda} \leq \liminf _{\lambda \uparrow 1} \Phi(\lambda)$.

3. Recall that the definition of $P_{\lambda}$ involved a parameter $\beta$. We now adopt the notations $P_{\lambda}^{(\beta)}, w_{\lambda}^{(\beta)}$ to indicate the dependence of $P_{\lambda}, w_{\lambda}$ on $\beta$. Set $a=D_{W_{\alpha}}(v)$, where $v$ is the function in $\operatorname{HBD}\left(W_{\alpha} ; \partial W_{\alpha}\right)$ determined by $v\left(p^{*}\right)=1$.

Lemma. Let $b, c$ be given such that $a<b<c$. It is possible to choose $\beta \in(0, \alpha / 2), \lambda \in(0,1)$ such that

$$
\begin{aligned}
& D_{W_{\alpha}}\left(w_{\lambda}^{(\beta)}\right)<b, \\
& E_{W_{\alpha}}^{P_{\lambda}^{(\beta)}}\left(w_{\lambda}^{(\beta)}\right)=c .
\end{aligned}
$$

Note that for $\beta \leq \beta^{\prime}$ we have $P_{\lambda}^{(\beta)} \leq P_{\lambda}^{\left(\beta^{\prime}\right)}$ and that $\lim _{\beta \downarrow 0} P_{\lambda}^{(\beta)}=0$. Thus in view of (4), (5) we have

$$
\lim _{\beta \downarrow 0}\langle 1,1\rangle_{W_{\alpha}}^{P_{1}^{(\beta)}}=0, \lim _{\beta \downarrow 0} \int_{W_{\alpha}} P_{1 / 2}^{(\beta)}=0 .
$$

We therefore may choose $\beta$ such that

$$
\langle 1,1\rangle_{W_{\alpha}^{(\beta)}}^{P_{\alpha}^{(\beta)}}<\frac{b-a}{2}
$$


and

$$
\int_{W_{\alpha}} P_{1 / 2}^{(\beta)}<\frac{b-a}{2} .
$$

For any $\lambda \in(0,1]$ we have $T_{D, W_{\alpha}} w_{\lambda}^{(\beta)}=v$ and hence

$$
\begin{aligned}
D_{W_{\alpha}}\left(w_{\lambda}^{(\beta)}\right) & =D_{W_{\lambda}}(v)+\left\langle w_{\lambda}^{(\beta)}, w_{\lambda}^{(\beta)}\right\rangle_{W_{\alpha}}^{P^{(\beta)}} \\
& \leq a+\langle 1,1\rangle_{W_{\alpha}}^{P^{(\beta)}}<\frac{a+b}{2},
\end{aligned}
$$

which shows that (7) holds for this $\beta$ and any $\lambda \in(0,1]$. By this and (9) we obtain

$$
E_{W_{\alpha}}^{P_{1 / 2}^{(\beta)}}\left(w_{1 / 2}^{(\beta)}\right)=D_{W_{\alpha}}\left(w_{1 / 2}^{(\beta)}\right)+\int_{W_{\alpha}}\left(w_{1 / 2}^{(\beta)}\right)^{2} P_{1 / 2}^{(\beta)}<\frac{a+b}{2}+\frac{b-a}{2}=b .
$$

In view of Lemma 2 we can choose $\lambda \in\left(\frac{1}{2}, 1\right)$ so that (8) also holds.

4. We use the notation $v_{\alpha}$ to indicate the dependence of the function $v \in H B D\left(W_{\alpha} ; \partial W_{\alpha}\right)$ with $v\left(p^{*}\right)=1$ on $\alpha$. We claim that

$$
D_{W_{\alpha}}\left(v_{\alpha}\right)=\frac{2 \pi}{\pi} \text {. }
$$

In fact, $v_{\alpha}\left|W_{\alpha}=1-\alpha^{-1} G_{T}\left(\cdot, q_{0}\right)\right| W_{\alpha}$ and hence (10) follows from the formula $D_{W_{\alpha}}\left(G_{T}\left(\cdot, q_{0}\right)\right)=2 \pi \alpha^{-1}$ (cf. [6]). Define $\alpha_{n}=4^{n+1} \pi, n=1,2, \ldots$ Then by (10) we have

$$
D_{W_{\alpha_{n}}}\left(v_{\alpha_{n}}\right)=\frac{1}{2 \cdot 4^{n}} .
$$

According to Lemma 3 we may choose $\lambda_{n}, \beta_{n}$ such that

$$
\delta_{n}=D_{W_{\alpha_{n}}}\left(w_{\lambda_{n}}^{\left(\beta_{n}\right)}\right)<\frac{1}{4^{n}}
$$

and

$$
\varepsilon_{n}=E_{W_{\alpha_{n}}}^{P_{\lambda_{n}}}\left(w_{\lambda_{n}}^{\left(\beta_{n}\right)}\right)=\frac{1}{2^{n}},
$$

for $n=1,2, \ldots$ Consider $W_{2 \alpha_{n}}=\left\{p \in T \mid r(p)>e^{-2 \alpha_{n}}\right\}$ and $v_{2 \alpha_{n}} \in$ $H B D\left(W_{2 \alpha_{n}} ; \partial W_{2 \alpha_{n}}\right)$ such that $v_{2 \alpha_{n}}\left(p_{n}^{*}\right)=1$. It can easily be seen that

$$
v_{2 \alpha_{n}} \mid \partial W_{\alpha_{n}}=\frac{1}{2} \text {. }
$$

We prepare infinitely many copies $T_{n}$ of $T, n=1,2, \ldots$ and view $W_{2 \alpha_{n}}$ as being a subsurface of $T_{n}$. Let $V=\mathbf{C} \backslash \cup_{1 \leq n<\infty}\{|z-3 n| \leq 1\}$. We weld $W_{2 \alpha_{n}}$ to $V$ by identifying $\partial W_{2 \alpha_{n}}$ with $\{|z-3 n|=1\}, n=1,2, \ldots$ and let 
$R$ be the resulting Riemann surface. We now view $W_{\alpha_{n}}, W_{2 \alpha_{n}}$ as subsurfaces of $R$ and denote them simply by $W_{n}, U_{n}$. We regard $v_{\alpha_{n}}, v_{2 \alpha_{n}}$ as being defined on $W_{n}, U_{n}$ and denote them by $v_{n}, u_{n}$. Let $\Delta$ be the harmonic boundary of $R$. Since $\operatorname{dim} H B D\left(W_{n} ; \partial W_{n}\right)=1, \bar{W}_{n} \cap \Delta$ consists of a single point $p_{n}^{*}$. Set $\Delta_{1}=\left\{p_{1}^{*}, p_{2}^{*}, \ldots\right\}$. The fact that $u_{n} \mid \partial W_{n}=\frac{1}{2}, n=$ $1,2, \ldots$, i.e. (14), implies $\bar{\Delta}_{1}=\Delta$ (cf. [3]). Let $W=\cup_{1 \leq n<\infty} W_{n}$ and define $v$ on $R$ by $v \mid W_{n}=v_{n}, n=1,2, \ldots$ and $v \mid R \backslash W=0$. Then by (11) we see that $v \in H B D(W ; \partial W)$. Since $v \mid \Delta_{1}=1$, we must have $v \mid \Delta=1$ and consequently $\bar{W} \backslash \partial \bar{W}$ is a neighborhood of $\Delta$ in $R^{*}$.

Define a 2 -form $P$ on $R$ by

$$
P \mid W_{n}=P_{\lambda_{n}}^{\left(\beta_{n}\right)}, n=1,2, \ldots \text { and } P \mid R \backslash W=0
$$

We view $w_{\lambda_{n}}^{\left(\beta_{n}\right)}$ as a function on $W_{n}$ and use the simplified notation $w_{n}$ for it. In this notation (12) and (13) are written as

$$
\begin{aligned}
& \delta_{n}=D_{W_{n}}\left(w_{n}\right)<\frac{1}{4^{n}}, \\
& \varepsilon_{n}=E_{W_{n}}^{P}\left(w_{n}\right)=\frac{1}{2^{n}},
\end{aligned}
$$

$n=1,2, \ldots$ For $X=D, E$ define measures $m^{P X}$ on $\Delta$ by setting $m^{P X}\left(\Delta \backslash \Delta_{1}\right)=0$ and

$$
m^{P D}\left(p_{n}^{*}\right)=\delta_{n}, m^{P E}\left(p_{n}^{*}\right)=\varepsilon_{n}
$$

$n=1,2, \ldots$ We denote the bounded continuous functions on $\Delta$ by $B(\Delta)$.

LEMMA. For $X=D$ or $E$

(i) $P B X(W ; \partial W) \mid \Delta=B(\Delta)$,

(ii) $P X(W ; \partial W) \mid \Delta=L^{2}\left(\Delta, m^{P X}\right)$.

Since (i) is an easy consequence of (ii) we proceed directly to the proof of (ii). We consider only the case $X=E$ as $X=D$ is analogous. Let $s \in P E(W ; \partial W)$. Then $+\infty>E_{W}^{P}(s)=\Sigma_{1}^{\infty} E_{W_{n}}^{P}(s)$. Recall that $\operatorname{PE}\left(W_{n} ; \partial W_{n}\right)$ is spanned by $w_{n}$. Thus $s \mid W_{n}=a_{n} w_{n}$ with $a_{n}=s\left(p_{n}^{*}\right)$. We see by (16) that $E_{W_{n}}^{P}(s)=a_{n}^{2} \varepsilon_{n}$ and hence $\left\{a_{n}\right\} \in L^{2}\left(\Delta, m^{P E}\right)$. Conversely, if $\left\{a_{n}\right\} \in L^{2}\left(\Delta, m^{P}\right)$, then by (16) the function $s=\Sigma_{1}^{\infty} a_{n} w_{n}$ is in $P E(W ; \partial W)$ with $s \mid \Delta=a_{n}, m^{P E}$-a.e. 
5. We arrive at our main result.

THEOREM. The 2-form $P$ and the Riemann surface $R$ have the property that

$$
\operatorname{PBE}(R)=P B D(R) \text { and } P E(R) \neq P D(R) .
$$

Since $\bar{W} \backslash \partial \bar{W}$ is a neighborhood of $\Delta$, we see that $\mu_{B D}$ is surjective (cf. [8]). By Theorem 1 we see that $\mu_{B D}^{P}$ and $\mu_{B E}^{P}$ are surjective as well. From Lemma 4(i) we deduce that $P B D(W ; \partial W)=P B E(W ; \partial W)$. Thus the mapping $\mu_{B D}^{P} \circ\left(\mu_{B E}^{P}\right)^{-1}: P B E(R) \rightarrow P B D(R)$ is a bijection and the first part of the assertion follows.

Let $f$ be defined on $\Delta_{1}$ by $f\left(p_{n}^{*}\right)=2^{n / 2}, n=1,2, \ldots$ By (15) and (16) we see that $f \in L^{2}\left(\Delta, m^{P D}\right)$ but $f \notin L^{2}\left(\Delta, m^{P E}\right)$. According to Lemma 4(ii) there is a function $s \in P D(W ; \partial W)$ such that $s \mid \Delta=f, m^{P D}$-a.e. Set $u=\mu_{D}^{P} s \in P D(R)$ and $h=T_{D} u$. By Theorem 1 we have $h \in$ $\mu_{D}(H D(W ; \partial W))$. If $u$ were in $P E(R)$, then in view of $h=T_{E} u$ Theorem 1 would imply that $u \in \mu_{E}^{P}(P E(W ; \partial W))$. But since $u \mid \Delta \notin L^{2}\left(\Delta, m^{P E}\right)$, Lemma 4(ii) rules out the possibility of $u$ being in $\mu_{E}^{P}(P E(W ; \partial W))$ and the assertion $u \notin P E(R)$ follows.

It is clear that there is a neighborhood $V^{*}$ of $\Delta$ with $\int_{V^{*} \cap R} P<+\infty$ but we have not been able to determine whether $\int_{R} P<+\infty$. Thus the relation between $\int_{R} P<+\infty$ and $P E(R)=P D(R)$ remains open.

\section{REFERENCES}

1. M. Glasner and R. Katz, On the behavior of solutions of $\Delta u=P u$ at the Royden boundary, J. Analyse Math., 22 (1969), 345-354.

2. M. Glasner and M. Nakai, Images of reduction operators, Archive Rat. Mech. Anal., 75 (1981), 387-406.

3. Roles of sets of nondensity points, Israel J. Math., 36 (1980), 1-12.

4. M. Nakai, Dirichlet finite solutions of $\Delta u=P u$ on open Riemann surfaces, Kōdai Math. Sem. Rep., 23 (1971), 385-397.

5. 67-87.

6. U_ Uniform densities on hyperbolic Riemann surfaces, Nagoya Math. J., 51 (1973), $1-24$.

7. Canonical isomorphisms of energy finite solutions of $\Delta u=P u$ on open Riemann surfaces, Nagoya Math. J., 56 (1975), 79-84.

8. _ Extremizations and Dirichlet integrals on Riemann surfaces, J. Math. Soc. Japan, 28 (1976), 581-603.

9. __ Malformed subregions of Riemann surfaces, J. Math. Soc. Japan, 29 (1977), 779-782.

10. __ An example on canonical isomorphism, Nagoya Math. J., 20 (1978), 25-40.

11. M. Nakai and S. Segawa, Tôki covering surfaces and their applications, J. Math. Soc. Japan, 30 (1978), 359-373. 
12. I. Singer, Boundary isomorphism between Dirichlet finite solutions of $\Delta u=P u$ and harmonic functions, Nagoya Math. J., 50 (1973), 7-20.

Received April 16, 1981. The second named author is supported by a Grant-in-Aid for Scientific Research, the Japanese Ministry of Education, Science and Culture.

Pennsylvania State University

UNIVERSITY PARK, PA 16802

U.S.A.

AND

NAgOYA INSTITUTE OF TECHNOLOGY

GoKiso, ShOWA, NAgoYa 466

JAPAN 



\title{
PACIFIC JOURNAL OF MATHEMATICS EDITORS
}

\author{
Donald BABBITT (Managing Editor) \\ University of California \\ Los Angeles, CA 90024 \\ Hugo Rossi \\ University of Utah \\ Salt Lake City, UT 84112 \\ C. C. Moore and Arthur Ogus \\ University of California \\ Berkeley, CA 94720
}

\author{
J. Dugundi \\ Department of Mathematics \\ University of Southern California \\ Los Angeles, CA 90089-1113
}

R. FinN and H. SAMELSON

Stanford University

Stanford, CA 94305

\section{ASSOCIATE EDITORS}
R. ARENS
E. F. BECKENBACH
B. H. NeumanN
F. WOLF
K. YoSHIDA (1906-1982)

\section{SUPPORTING INSTITUTIONS}

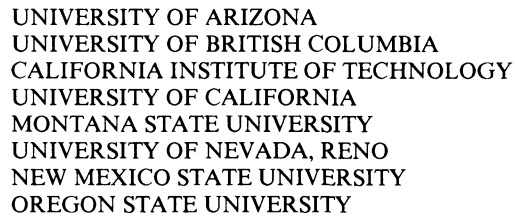

UNIVERSITY OF ARIZONA

UNIVERSITY OF BRITISH COLUMBIA

CALIFORNIA INSTITUTE OF TECHNOLOGY

UNIVERSITY OF CALIFORNIA

MONTANA STATE UNIVERSITY

UNIVERSITY OF NEVADA, RENO

NEW MEXICO STATE UNIVERSITY

OREGON STATE UNIVERSITY

\author{
UNIVERSITY OF OREGON \\ UNIVERSITY OF SOUTHERN CALIFORNIA \\ STANFORD UNIVERSITY \\ UNIVERSITY OF HAWAII \\ UNIVERSITY OF TOKYO \\ UNIVERSITY OF UTAH \\ WASHINGTON STATE UNIVERSITY \\ UNIVERSITY OF WASHINGTON
}

The Supporting Institutions listed above contribute to the cost of publication of this Journal, but they are not owners or publishers and have no responsibility for its content or policies.

Mathematical papers intended for publication in the Pacific Journal of Mathematics should be in typed form or offset-reproduced (not dittoed), double spaced with large margins. Please do not use built up fractions in the text of the manuscript. However, you may use them in the displayed equations. Underline Greek letters in red, German in green, and script in blue. The first paragraph must be capable of being used separately as a synopsis of the entire paper. In particular it should contain no bibliographic references. Please propose a heading for the odd numbered pages of less than 35 characters. Manuscripts, in triplicate, may be sent to any one of the editors. Please classify according to the scheme of Math. Reviews, Index to Vol. 39. Supply name and address of author to whom proofs should be sent. All other communications should be addressed to the managing editor, or Elaine Barth, University of California, Los Angeles, California 90024.

There are page-charges associated with articles appearing in the Pacific Journal of Mathematics. These charges are expected to be paid by the author's University, Government Agency or Company. If the author or authors do not have access to such Institutional support these charges are waived. Single authors will receive 50 free reprints; joint authors will receive a total of 100 free reprints. Additional copies may be obtained at cost in multiples of 50 .

The Pacific Journal of Mathematics is issued monthly as of January 1966. Regular subscription rate: $\$ 132.00$ a year (6 Vol., 12 issues). Special rate: $\$ 66.00$ a year to individual members of supporting institutions.

Subscriptions, orders for numbers issued in the last three calendar years, and changes of address should be sent to Pacific Journal of Mathematics, P.O. Box 969, Carmel Valley, CA 93924, U.S.A. Old back numbers obtainable from Kraus Periodicals Co., Route 100, Millwood, NY 10546.

The Pacific Journal of Mathematics ISSN 0030-8730 is published monthly by the Pacific Journal of Mathematics at P.O. Box 969, Carmel Valley, CA 93924. Application to mail at Second-class postage rates is pending at Carmel Valley, California, and additional mailing offices. Postmaster: Send address changes to Pacific Journal of Mathematics, P. O. Box 969, Carmel Valley, CA 93924.

PUBLISHED BY PACIFIC JOURNAL OF MATHEMATICS, A NON-PROFIT CORPORATION

Copyright $\odot 1983$ by Pacific Journal of Mathematics 


\section{Pacific Journal of Mathematics}

Vol. 104, No. $2 \quad$ June, 1983

Leo James Alex, Simple groups and a Diophantine equation ........... 257

Herbert James Alexander and John Wermer, On the approximation of

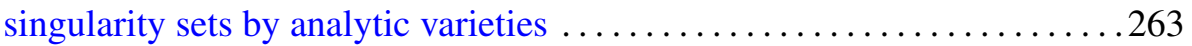

Waleed A. Al-Salam and Mourad Ismail, Orthogonal polynomials associated with the Rogers-Ramanujan continued fraction .......... 269

J. L. Brenner and Roger Conant Lyndon, Permutations and cubic

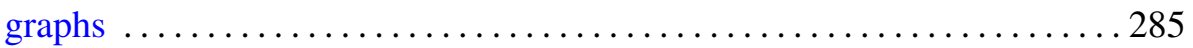

Ian George Craw and Susan Ross, Separable algebras over a commutative

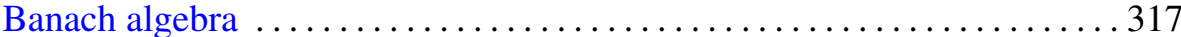

Jesus M. Dominguez, Non-Archimedean Gel'fand theory ............. 337

David Downing and Barry Turett, Some properties of the characteristic of

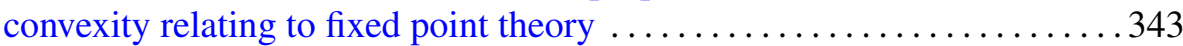

James Arthur Gerhard and Mario Petrich, Word problems for free objects in certain varieties of completely regular semigroups $\ldots \ldots \ldots 351$

Moses Glasner and Mitsuru Nakai, Surjective extension of the reduction

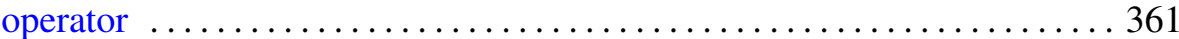

Takesi Isiwata, Ultrafilters and mappings $\ldots \ldots \ldots \ldots \ldots \ldots \ldots \ldots \ldots \ldots \ldots$

Lowell Duane Loveland, Double tangent ball embeddings of curves in $E^{3}$

Douglas C. McMahon and Ta-Sun Wu, Homomorphisms of minimal flows

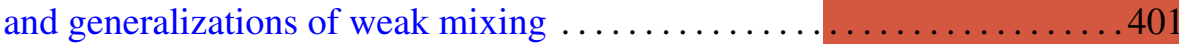

P. H. Maserick, Applications of differentiation of $\mathscr{L}_{p}$-functions to semilattices

Wayne Bruce Powell and Constantine Tsinakis, Free products in the class of abelian $l$-groups

Bruce Reznick, Some inequalities for products of power sums

C. Ray Rosentrater, Compact operators and derivations induced by

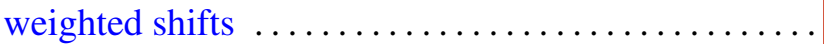

Edward Silverman, Basic calculus of variations

Charles Andrew Swanson, Criteria for oscillatory sublinear Schrödinger equations

David J. Winter, The Jacobson descent theorem 